\title{
Target Classification in Sparse Sampling Acoustic Sensor Networks Using IDDC Algorithm*
}

\author{
Youngsoo Kim, Daeyoung Kim, Taehong Kim, Jongwoo Sung, and Seongeun Yoo \\ Real-time and Embedded Systems Laboratory, \\ Information and Communications University (ICU), \\ 119 Munjiro, Yuseong-Gu, Daejon, Korea, Postal Code: 305-714, \\ Phone: +82-42-866-6811; Fax: +82-42-866-6810 \\ \{pineland, kimd, damiano, jwsung, seyoo\}@icu.ac.kr
}

\begin{abstract}
The analysis of time series using data mining techniques can be effective when all targets have their own inherent patterns in a sparse sampling acoustic sensor network where no valid feature of frequency can be extracted. However, both problems of local time shifting and spatial variations should be solved to deploy the time series analysis. This paper presents time-warped similarity measure algorithms in order to solve the two problems through time series, and we propose the IDDC (Improved Derivative DTW-Cosine) algorithm to deliver the optimal result and prove the performance with some experiments. The experimental results show that the object classification accuracy rate of the proposed algorithm outperforms the other time-warped similarity measure algorithms by at least $10.23 \%$. Since this proposed algorithm produces such a satisfactory result with sparse sampling data, it allows us to classify objects with relatively low overhead.
\end{abstract}

\section{Introduction}

The target classification using sparsely sampled data is one of the key issues of a Wireless Sensor Network (WSN) application since it consists of a large number of low-power and inexpensive sensor nodes. When distributed sensor nodes sense and transfer data to a base station (BS) through WSN, the network cost might increase dramatically as the hop count increases. Especially in the case of acoustic data, while it is one of the most frequently used and informative sensors in a target classification system, it is so complicated and variable that it needs more numerous and dense data to obtain sufficient information. The bigger the WSN is, the less data should be basically transferred to increase the life span. It is because the mechanism of sensing frequently and transferring all of the data causes the nodes to be exhausted very fast.

Most of the existing research [1][2][3] for classification in acoustic WSNs have extracted features using the FFT and classified targets with some classification

\footnotetext{
* This research was supported by the MIC (Ministry of Information and Communication), Korea, under the ITRC (Information Technology Research Center) support program supervised by the IITA (Institute of Information Technology Advancement)(IITA-2006C1090-0603-0015) and the Korea Science and Engineering Foundation (KOSEF) grant funded by the Korea government(MOST) (No. R0A-2007-000-10038-0).
} 
algorithms such as k-nearest neighbor $(\mathrm{kNN})$, maximum likelihood (ML), support vector machine (SVM), etc. However, frequency analysis typically need a high computational cost as well as a high sampling (or sensing) rate to affirm the performance and thus, a broader bandwidth network to transfer. That is why a new approach is needed in the area of sparse frequency. When all targets have their own inherent patterns, time series analysis can be an effective method for target classification with sparse sampling data in acoustic WSNs. A lot of research have been performed for this manner, mainly to retrieve some patterns in a large database or to do data mining [5][6][7][8][9]. However, the problem of time shifting and the problem of spatial variations caused by the strength of volume over different distances should be solved to apply the manner to WSNs.

In this paper, we assume the innate pattern of each target could be found, and we focus on target classification with the patterns using the Improved Derivative DTWCosine (IDDC) algorithm proposed as a new technique. Our data set are made by adding various effects to each original signal to emulate some distortion effects, and the performance is analyzed over volumes to consider the spatial variations. We first do preprocessing all data and make the reference model using the PAA (Piecewise Aggregate Approximation) [7] which draws the contour of each target. The input signal array is then used to get a similarity (correlation) for each reference by the proposed algorithm. Finally, the weak performances in lower and higher volumes are improved using a smoothing technique.

The rest of this paper is organized as follows. Section 2 contains a discussion of characteristics of acoustic signal in a sparse sampling WSN, and section 3 describes our algorithms. Our data collection and experimental setup are illustrated, and the performance of proposed algorithm is empirically compared in section 5. Finally, Section 6 concludes our experiments and discusses for future works.

\section{Characteristics of Acoustic Signal in a Sparse Sampling WSN}

We can see how much information of frequency remain in a sparse sampling WSN through looking into the spectrogram. The sparser sampled, the more smoothed with respect to frequency in the spectrogram of signal. Fig. 1 shows an example of aggravated spectrogram caused by sparse sampling. It is shown to be plain with respect to frequency, which means little frequency information is contained in the signal as a result of sparse sampling.

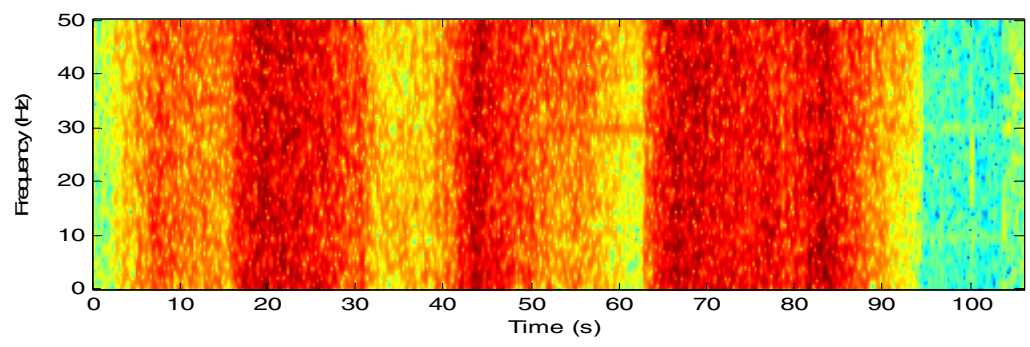

Fig. 1. Spectrogram of airplane signal sampled at $100 \mathrm{~Hz}$ with 80 points-STFT (Short-Time Fourier Transform), Hamming window and 90\% overlap 
As shown in Fig. 2, the shape of sampled signal can be different despite of being generated by the same target whenever sampled. It is because the sampling point of each signal differs from the others, which is one of the characteristics of WSN to make it more difficult to classify. To reduce the complexity of the signals, they are preprocessed as will be discussed in Sec 4.1. In addition, each signal not only is shifted with respect to time axis but also has different strength associated with the distance between the sensor node and the target in the field of WSN. So, we need a new algorithm which can solve the above problems efficiently.

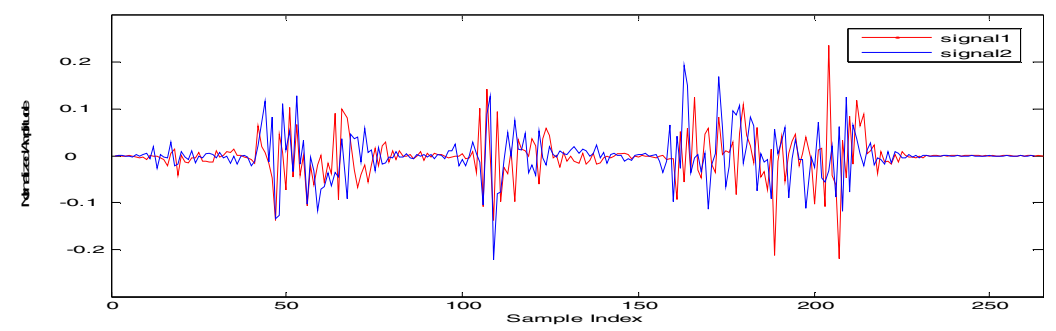

Fig. 2. Comparing two sampled signals made by different sampling points for the signal of airplane. Their shapes are different with each other.

\section{Improved Derivative DTW-Cosine (IDDC) Algorithm}

There are two problems, local time shifting and spatial variations, in applying the time series manner to the classification system of WSN. The first problem arises when a sequence is shifted or has different lengths from the other. It is solved by a time warping algorithm which uses dynamic programming. The other problem is caused by distance, which means that all the signals from the same object should be identified regardless of the strength of volume.

\subsection{Time-Warped Algorithms}

Three algorithms namely DTW [4], ED [5] and LCS [9] can be typically applied as a time warping algorithm. Table 1 shows comparing the core parts, distance function, of these algorithms. The DTW algorithm is frequently used to find the warped path through a matrix of points representing possible time alignments between two patterns. Given two time sequences, it fills an $m$ by $n$ matrix representing the distances of the best possible partial path using a recursive formula as its distance function in Table 1. The alignment that results in the minimum distance between the two sequences has value $D(m, n)$. To solve the problem of time scaling in time series, the DTW aligns the time axis and easily produces the matched array of time series in a well aligned manner. The ED, also known as the Levenshtein distance, between two strings is to find the minimum number of operations needed to transform one string into the other, where an operation is an insertion, deletion, or substitution. Lastly, the LCS finds the longest subsequence that two sequences have in common, regardless of the length and the number of intermittent mismatching symbols. However, the 
performances of the LCS and the ED depend heavily on correct setting of the scaling threshold, which may be a particularly difficult problem for some applications as well as in WSNs.

Table 1. Comparing the time warping algorithms

\begin{tabular}{c|c}
\hline Name & Distance function \\
\hline Dynamic Time Warping (DTW) & $D(i, j)=d(i, j)+\min \left\{\begin{array}{l}D(i, j-1) \\
D(i-1, j) \\
D(i-1, j-1)\end{array}\right.$ \\
\hline Edit Distance (ED) & $d\left(x_{i}, y_{i}\right)=\left\{\begin{array}{cc}0 & \text { if } x_{i}=y_{i} \\
1 & \text { if } x_{i} \text { or } y_{i} \text { is a gap } \\
1 & \text { otherwise }\end{array}\right.$ \\
\hline $\begin{array}{c}\text { Longest Common Sub-sequence } \\
\text { (LCS) }\end{array}$ & $d(i, j)=\left\{\begin{array}{c}0, \quad \text { if } \mathrm{i}=0 \text { or } \mathrm{j}=0 \\
d(i-1, j-1)+1, \text { if } \mathrm{i}, \mathrm{j}>0 \text { \& } \mathrm{x}_{\mathrm{i}}=\mathrm{y}_{\mathrm{j}} \\
\max [d(i, j-1), d(i-1, j)], \\
\text { otherwise }\end{array}\right.$ \\
\hline
\end{tabular}

\subsection{Similarity Measure Algorithms}

The similarity measure algorithm is to measure the similarity settling the problem of spatial variations caused by distance. All signals from an object should be identified regardless of the strength of volume. The similarity can be measured with the degree of correlation or distance between two sequences. It can be reflected by the Euclidean, the Pearson [11], or the Cosine correlation algorithms described in Table 2 assuming that $\mathrm{x}^{\prime}$ and $\mathrm{y}^{\prime}$ is a matched array respectively.

Table 2. Comparing the correlation (similarity) measure algorithms

\begin{tabular}{c|c}
\hline Name & Distance function \\
\hline Euclidean & $C\left(x^{\prime}, y^{\prime}\right)=\sqrt{\sum_{i=1}^{N}\left(x_{i}^{\prime}-y_{i}^{\prime}\right)^{2}}$ \\
\hline Pearson & $C\left(x^{\prime}, y^{\prime}\right)=\frac{\sum_{i=1}^{N}\left(x_{i}^{\prime}-m_{x}\right)\left(y_{i}^{\prime}-m_{y}\right)}{\sqrt{\left[\sum_{i=1}^{N}\left(x_{i}^{\prime}-m_{x}\right)^{2}\right]\left[\sum_{i=1}^{N}\left(y_{i}^{\prime}-m_{y}\right)^{2}\right]}}$ \\
\hline Cosine & $C\left(x^{\prime}, y^{\prime}\right)=\frac{\frac{1}{N} \sum_{i=1}^{N} x_{i}^{\prime} \times y_{i}^{\prime}}{\left\|x^{\prime}\right\| \times\left\|^{\prime}\right\|}$ \\
\hline
\end{tabular}

To compare these algorithms with each other in WSN applications, suppose that there are three signals which have been collected on a BS in a WSN as shown in Fig. 3. y1 and y2 can happen when a moving object is varying in distance to sensor 
nodes over time, which means a similarity measure algorithm should identify them. Referring to Table 3, the measures by the Euclidean algorithm represent distance values while the Cosine and the Pearson algorithms compute similarity. The more similar the signals are, the less is the Euclidean distance and the larger the measure of the Cosine and the Pearson similarities. The Pearson and the Cosine similarity algorithms identify y1 and y2 while the Euclidean algorithm can not classify them well. On the other hand, y3 and the others may not be caused by the same object, which means they can be regarded as not exactly the same but similar. Table 3 shows that the Cosine similarity identifies minute differences while the Pearson similarity does not. An original signal should not be confused with the others to maintain the performance. That is why the former outperforms the latter. Consequently, we can say that the Cosine similarity can represent the characteristics of signal in WSN better than the others, which will be discussed in more details in Sec. 4.2.

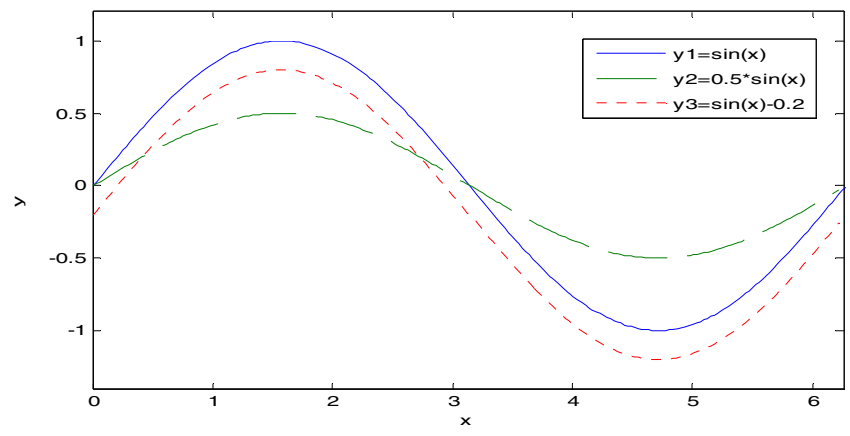

Fig. 3. Three signals that could occur in a WSN field

Table 3. The distance/correlation measures of three algorithms

\begin{tabular}{c|ccc}
\hline Algorithm & Euclidean & Pearson & Cosine \\
\hline $\mathrm{y} 1 \leftrightarrow \mathrm{y} 2$ & 2.8025 & 1.0000 & 1.0000 \\
$\mathrm{y} 2 \leftrightarrow \mathrm{y} 3$ & 1.5875 & 1.0000 & 0.9622 \\
$\mathrm{y} 3 \leftrightarrow \mathrm{y} 1$ & 3.2211 & 1.0000 & 0.9622 \\
\hline
\end{tabular}

\subsection{Improved Derivative DTW-Cosine (IDDC) Algorithm}

The IDDC algorithm combines the derivative DTW (DDTW) among variants of the DTW with the Cosine algorithm as the best classifier. The classic DTW algorithm has a tendency of producing unreasonable alignments where a single point in one sequence is mapped onto a large subsection of the other sequence when making a matching array (see the (a) of Fig. 4). It is because the algorithm finds an optimal path considering only the distance between a point of one sequence and its corresponding points of the other sequence. The problem led E. Keogh [6] to perform the DTW on the derivative of the time series instead of on the sequence itself. The (a) and (b) of Fig. 4 is to compare the results of the DTW and the DDTW. The DTW seems to fail to find the optimal alignment while the alignments produced by the DDTW look 
better. However, the DDTW has a tendency of being more sensitive to noise than the DTW. So, E. Keogh [6] suggested the following estimate for robustness to outliers through simplicity and generality.

$$
Q_{I}=\frac{\left(q_{i}-q_{i-1}\right)+\left(\left(q_{i+1}-q_{i-1}\right) / 2\right)}{2}, \quad 1<i<m .
$$

where $q_{i}$ is a point of a sequence. Assume that the average $m_{N}$ and the standard deviation $\sigma_{N}$ of background noise are known, the estimate $Q$ could be regarded as zero when $q \leq\left(m_{N}+\sigma_{N}\right)$ to avoid the influence of noise. The noise of background is mostly close to zero. In addition, the diagonal path $d(m-1, n-1)$ should be given the priority among the next paths not to lose the correct path when the next path will be found as described in (c) and (d) of Fig. 4. According to our experiments, this scheme should be considered especially for matching the points of background sounds. Finally, the Cosine algorithm measures the similarity with the array which this improved derivative DTW produced.

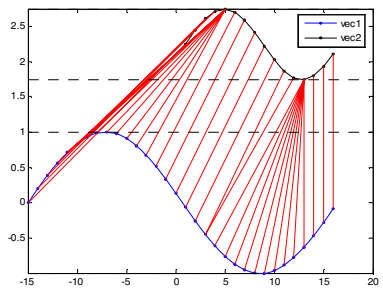

(a) DTW

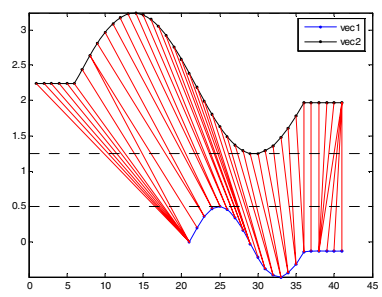

(c) DDTW (ii)

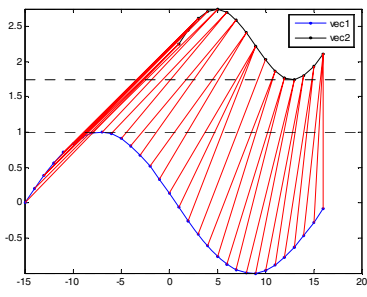

(b) DDTW(i)

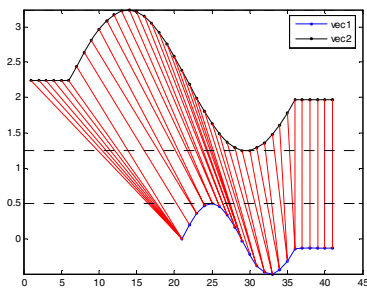

(d) Improved DDTW

Fig. 4. Comparing the DTW, the DDTW, and the improved DDTW

\section{Experiments and Evaluations}

We first describe our experimental setup briefly, and the performances of the ED, the LCS, the DTW, and the IDDC are compared with each other. We then show the effectiveness of similarity measure algorithms through several experiments of the time-warped similarity measure algorithms including a smoothing technique. 


\subsection{Data Collection and Experimental Setup}

Three types of military objects, airplane, tank and soldier, in Fig. 5 are classified in the experiment. As shown, the sound of a soldier is a step sound which is very periodic and the duration of local frame is very short while the sound of an airplane is sleek a little and has a long local frame. The tank makes the sound of irregular explosions against a background of the sound of engine and wheels, which has a monotonous energy. We added some effects and noise which produces some distortion to the signals, e.g. various Doppler effects, some hissing noises by size, echo, flanger, mechanize, pitch change, some volume transforming effects(fade in/out), and time warping. We made 31 test data per object and totally have 93 test data. Each file is sampled sparsely at $10 \mathrm{~Hz} 20$ times and classified 1860 times against objects before obtaining the result.
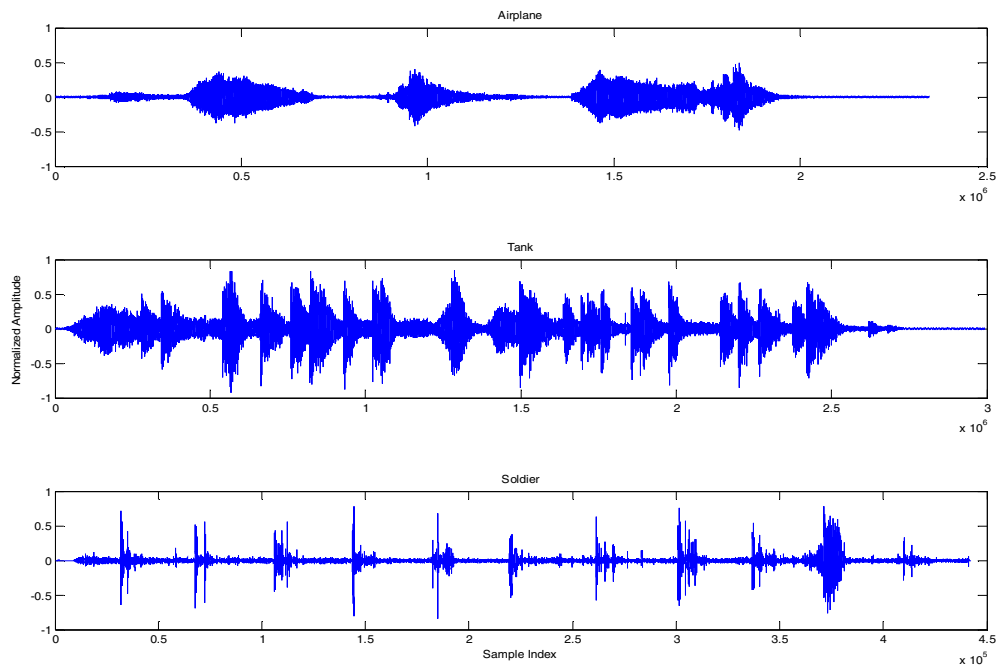

Fig. 5. Assumed signal patterns of military targets which can be occurred in a field of WSN

The overall experimental system architecture consists of preprocessing, making reference, and classification parts. First, in the preprocessing part, input signals are scaled between -1 and 1 , and its absolute values are taken to reduce the variation and the computational complexity. Fortunately since they constitute the piecewise power values of symmetry at each point and are highly correlated with each other, the absolute values are very efficient without losing any information. Also, the smoothing technique is used to improve the accuracy in higher and lower volumes in this paper. It is computed by Eq. 2 assuming that $N$ is the number of elements to smooth.

$$
x_{i}{ }^{\prime}=\frac{1}{N} \sum_{j=i+1}^{i+N} x_{j} .
$$


Second, to model the outline of the reference against the input signal, each reference signal is compressed to the length of input signal using the PAA technique, Keogh et al. [7] proposed, because each input has a different length case by case. The PAA compresses or models a signal as it draws the contour of the reference signal of an object as follows: Let $N$ be the dimensionality of the transformed time series we wish to work with $(1 \leq N \leq n)$. The $i$ th element of is calculated by Eq. 3. Lastly, the signal is classified to the object category which has the best similarity caused by the time-warped similarity measure algorithm and the decision rule.

$$
\overline{x_{i}}=\frac{N}{n} \sum_{j=\frac{n}{N}(i-1)+1}^{\frac{n}{N} i} x_{j} .
$$

\subsection{Comparison of Time Warping Algorithms}

Before comparing the effectiveness of the three time warping algorithms, the ED, the LCS and the DTW, we experimented with the performance of the ED and the LCS with three levels of volume - half $(-6.02 \mathrm{~dB})$, normal and double $(6.02 \mathrm{~dB})$ as shown Table 4. This is because the ED and the LCS should use a scaling threshold to apply to time series data, which consist of numeric values, and compare with the DTW. We explored the performance by varying the value of the threshold from 0.02 to 0.2 . As shown in Table 4, the optimal threshold has a tendency to move following the level of volume. i.e., the threshold shifts to a smaller value in higher volume while it becomes larger in lower volume. While the optimal threshold comes to be 0.06 in double volume, it is 0.08 and 0.1 in normal and half volume respectively. Consequently, it is clear that the threshold cannot be easily established over volume.

Table 4. Optimal threshold of ED and LCS

\begin{tabular}{c|cc}
\hline Volume level & ED & LCS \\
\hline Double Volume (6dB) & 0.07 & 0.06 \\
Normal Volume (0dB) & 0.08 & 0.08 \\
Half Volume (-6dB) & 0.1 & 0.1 \\
\hline
\end{tabular}

The Fig. 6 shows the comparison of the performance of the time warping algorithms including the DDTW as well as the Improved DDTW (IDDTW). All of the DTW, the DDTW, and the IDDTW give a similar performance with the optimal accuracy of the ED and the LCS in normal and high volume and all of them have a poor performance in lower volume. It means that an algorithm which can improve the performance, especially in the case of lower volume, is needed. The IDDTW can not only produce a matching array with ease but is also superior to the DTW and the DDTW over all volumes. Consequently, it is reasonable that any similarity measure algorithm should be combined with the IDDTW to get the highest performance. 


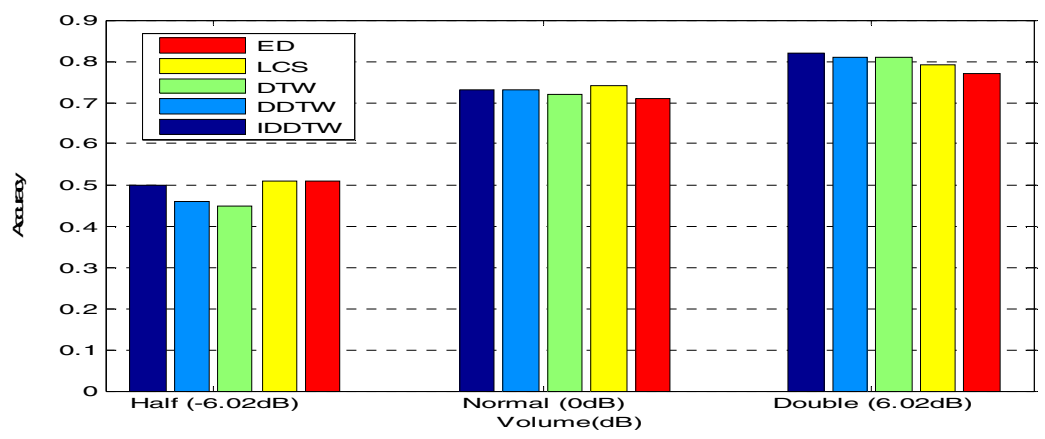

Fig. 6. Comparison of time warping algorithms

\subsection{Comparison of Time-Warped Similarity Measure Algorithms Based on the IDDTW}

We tested the IDDTW combined with all similarity measure algorithms as mentioned in section 3, and we found that the IDDC (IDDTW+Cosine) algorithm improves the performance in the area of lower volume very effectively and outperforms the other algorithms as depicted in Fig. 7. Although the performance of the IDDTW and the IDDE (IDDTW+Euclidean) algorithm shows a good performance from 5 to $8 \mathrm{~dB}$, their performances are poor in lower volumes as well as have a tendency of degrading dramatically after $8 \mathrm{~dB}$, which means they are highly dependent on the level of volume. On the other hand, the IDDP (IDDTW+Pearson) and the IDDC have saliently better accuracies in lower volume and are comparatively less affected by volume.

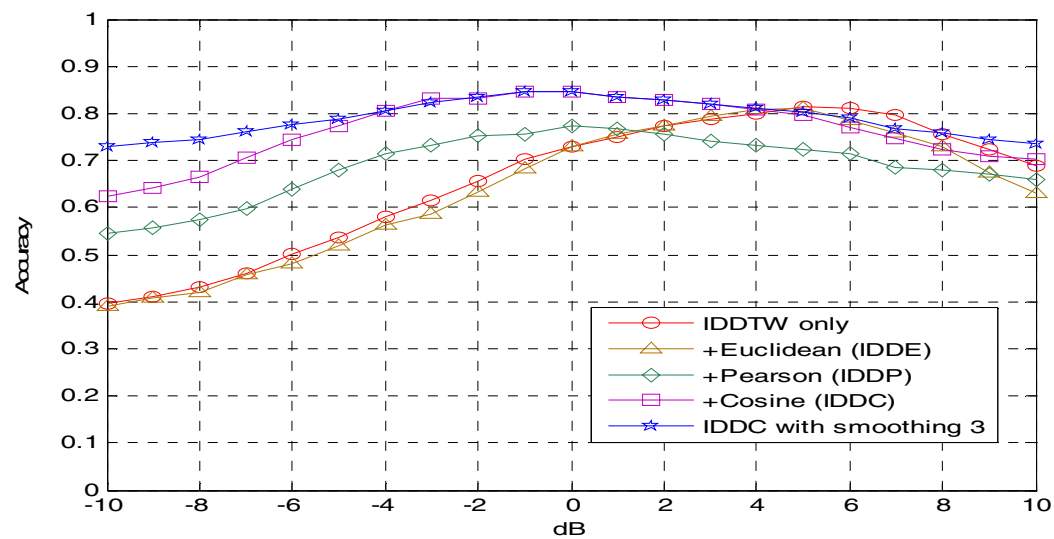

Fig. 7. Comparison of time-warped similarity measure algorithms

We experimented the IDDC algorithm + Gaussian smoothing which is helpful in improving the accuracy through avoiding distortions caused by odd elements. We 
found the optimal number of elements for smoothing should be 3 through several experiments, so that the 3 elements came to be applied for smoothing in Fig. 7. Anyway, it turned out to be effective in improving the accuracy in both lower and higher volumes.

Table 5 shows the confusion matrix of targets corresponding to volumes. The accuracies of tank and airplane outperform soldier by and large because the width of pillars of signal from the soldier are so narrow that they are often unable to be sampled while the interval between them are so large that even skipping sampling once can be critical to the performance. The influence of volume is also shown to depend on the sort of sound. i.e. monotonous signals such as an airplane is affected negatively by strong volume while complicated and variable signals such as a tank is positively affected. It is because more monotonous signal is modeled relatively better in lower volumes than in higher volumes. Consequently Considering a range from -10 $\mathrm{dB}$ to $10 \mathrm{~dB}$, the accuracy of the IDDTW is $65.35 \%$, the IDDE is $63.77 \%$, IDDP is $68.75 \%$, the IDDC algorithm is $76.55 \%$, and the IDDC algorithm with the smoothing technique $78.98 \%$ on the average. It means the optimized IDDC algorithm outperforms the other algorithms by at least $10.23 \%$.

Table. 5. Confusion matrix by the IDDC algorithm with the smooth 3 technique corresponding to volumes

\begin{tabular}{c|c|c|c|c}
\hline $\begin{array}{c}\text { Classified } \\
\text { Object }\end{array}$ & Volume & Soldier & Tank & Airplane \\
\hline \multirow{3}{*}{ Soldier } & Half & $\mathbf{0 . 6 1 3 3 5}$ & 0.23832 & 0.14833 \\
& Normal & $\mathbf{0 . 6 3 7 4 8}$ & 0.20700 & 0.15552 \\
& Double & $\mathbf{0 . 5 2 3 8 3}$ & 0.32384 & 0.15233 \\
\hline \multirow{3}{*}{ Tank } & Half & 0.09468 & $\mathbf{0 . 7 6 4 8 8}$ & 0.14044 \\
& Normal & 0.01512 & $\mathbf{0 . 9 6 2 0 5}$ & 0.02283 \\
& Double & 0.00463 & $\mathbf{0 . 9 9 0 7 5}$ & 0.00462 \\
\hline \multirow{3}{*}{ Airplane } & Half & 0.01600 & 0.03681 & $\mathbf{0 . 9 4 7 5 9}$ \\
& Normal & 0.00636 & 0.05602 & $\mathbf{0 . 9 3 7 6 2}$ \\
& Double & 0.00512 & 0.14042 & $\mathbf{0 . 8 5 4 4 6}$ \\
\hline
\end{tabular}

\section{Conclusion and Future Works}

We described the characteristics of acoustic signals in a sparse sampling WSN and proposed the IDDC algorithm as the best classifier algorithm of time series. Since the acoustic signals not only comprise dense positive and negative values, but they also constitute the piecewise power values of symmetry at each point and highly correlated with each other, their absolute values and the smoothing technique are taken to improve the accuracy. Even though the experimental data are made artificially, it makes sense that the proposed algorithm has a satisfactory accuracy over volumes and outperforms compared to the other time-warped algorithms by at least $10.23 \%$ on the whole. We can also infer that the method of time series analysis can work collaboratively with the method of frequency analysis to operate a WSN economically. So, it is sure that our work could be a baseline for the research of target classification using the time series approach in the future. 
Our future works will focus on applying physical features, the ZCR (Zero Crossing Rate), energy, etc, and multi-modal fusion to improve the accuracy since objects have different signatures from each other corresponding to multiple modalities, e.g. magnetic and seismic. The HMM (Hidden Markov Model) could also give us the capability to analyze more diverse, more general and longer signals.

\section{References}

1. Li, D., Wong, K.D., Hu, Y.H., Sayeed, A.M.: Detection, Classification and Tracking of Targets in Distributed Sensor Networks. IEEE Signal Processing Magazine, 17-29 (2002)

2. Meesookho, C., Narayanan, S., Raghavendra, C.S.: Collaborative classification applications in sensor networks. In: Second IEEE Sensor Array and Multichannel Signal Processing Workshop, pp. 370-374 (2002)

3. Duarte, M.F., Hu, Y.H.: Vehicle Classification in Distributed Sensor Networks. Journal of Parallel and Distributed Computing 64(7), 826-838 (2004)

4. Sakeo, H., Chiba, S.: Dynamic programming algorithm optimization for spoken word recognition. IEEE Trans. On Acoustics, Speech and Signal Processing 26, $43-49$ (1978)

5. Chen, L., Ng, R.: On the Marriage of Lp-Norm and. Edit Distance. VLDB, 792-803 (2004)

6. Keogh, E., Pazzani, M.: Derivative Dynamic Time Warping. In: The First SIAM International Conference on Data Mining (2001)

7. Keogh, E., Chakrabarti, K., Pazzani, M., Mehrotra, S.: Dimensionality Reduction for Fast Similarity Search in Large Time Series Databases. Knowledge and Information Systems Journal 3(3), 263-286 (2003)

8. Latecki, L.J., Megalooikonomou, V., Wang, Q., LakÄamper, R., Ratanamahatana, C., Keogh, E.: Partial Elastic Matching of Time Series. In: Proc. of 5th International Conference on Data Mining, pp. 701-704 (2005)

9. Guo, A., Siegelmann, H.T.: Time-Warped Longest Common Subsequence Algorithm for Music Retrieval. In: IS-MIR. International Conference on Music Information Retrieval (2004)

10. Chen, L., Äozsu, M.T., Oria, V.: Robust and Fast Similarity Search for Moving Object Trajectories. In: ACM SIGMOD international conference on Management of data, pp. 491-502 (2005)

11. Resnick, et al.: GroupLens: An Open Architecture for Collaborative Filtering of Netnews. In: Proceeding of CSCW 1994 (October 1994) 